\title{
Scar Selection in an Optical Fibre
}

\author{
V. Doya*, O. Legrand, C. Michel and F. Mortessagne
}

Laboratoire de Physique de la Matière Condensée, Université de Nice, France

\begin{abstract}
A multimode optical fiber with a D-shaped cross-section has already been proved to constitute a privileged system to image wave functions of a chaotic system. Nevertheless, diffraction of light at the fiber input precludes the excitation of individual wave functions and particularly the scar modes we want to investigate. We show that a mechanism of scar modes selection can be induced by the introduction of a localized gain.
\end{abstract}

PACS numbers: 05.45.Mt, 42.25.Dd, 42.81.-i

\section{Introduction}

Since their numerical evidence and their explanation by Heller [1] as "... extra density surrounding periodic orbits...", scar wave functions have been the subject of a great number of investigations. Different theoretical explanations of these singular wave functions have been proposed [2-4] and experimental investigations have been developed in parallel with the emergence of wave chaos experiments [5]. Since 1980, scar wave functions have been observed in various systems with different nature of waves, such as billiard wave functions of a chaotic microwave cavity [6] or recently remarkable patterns in surface waves [7]. A nice and evident way to image the wave functions of a chaotic cavity bases on using optical waves. To the contrary to microwave or elastodynamical experiments, experiments with optical waves offer a direct visualization of the wave functions. One of the first experiment with optical waves invoking the properties of classical chaos to explain the behavior of waves has been proposed by the group of Stone [8]. Until 2002, no direct observation of the wave functions of a chaotic optical billiard had been realized. Using the formal analogy between the Helmholtz equation governing the propagation of optical waves and the Schrödinger equation, we have then proposed an experiment of wave chaos in an optical fiber. The experiment, simple in its concept, offers a direct access to the spatial distribution of intensity by imaging the near-field intensity at the fiber output. Using a multimode fiber with a truncated transverse cross-section, we observed the scarring effect for the first time in an optical system [9]. The observed pattern was associated to the superposition of

*corresponding author; e-mail: valerie.doya@unice.fr 
a scar mode with a few generic ergodic modes [10] due to the diffraction at the input of the fiber that prevents the excitation of individual modes. With the aim to demonstrate that optical fiber can also constitute an ideal system to investigate pure scar modes, we propose to exploit the properties of the introduction of a gain medium. In this paper, we show that an appropriate location of the gain medium can induce a selective amplification of scar modes. This paper is organized as follows: in Sect. 2, the experimental setup is described and its relevance in the field of wave chaos is presented. Our main results about the experimental observation of the typical behavior of wave functions in a chaotic billiard are reported in Sect. 2.2. In order to investigate the scar wave functions, we have developed a selective excitation of scar by the way of optical amplification. These recent studies are described in Sect. 3.

\section{Presentation of the experimental system}

\subsection{Description of the system}

The multimode optical fiber used for this work has been designed and fabricated in our lab. The transverse cross-section of the fiber is a truncated disk fabricated from a $1 \mathrm{~cm}$ diameter preform of silica that has been cut at half its radius and pulled at a temperature low enough to maintain the D-shaped cross-section. The final dimensions of the fiber after pulling are $120 \mu \mathrm{m}$ for the diameter and $90 \mu \mathrm{m}$ for the truncated diameter (see Fig. 1). These dimensions differ from

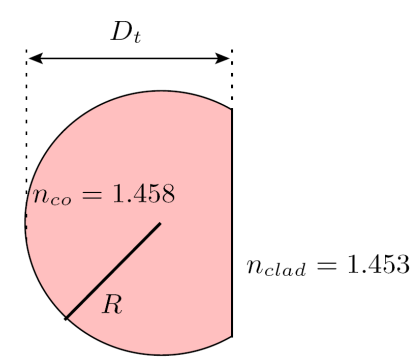

Fig. 1. Characteristical parameters of the chaotic fiber.

the typical $10 \mu \mathrm{m}$ diameter of a single mode optical fiber used in the field of optical telecommunication. The index of refraction of the silica core $n_{\text {co }}$ at our working wavelength is around 1.458. It is surrounded by a black silicon cladding with an index of refraction equal to $n_{\text {clad }}=1.453$. As the characteristic dimensions of the fiber are large compared to the optical wavelength, a collimated light beam that propagates along the fiber can be approximated, in the geometrical limit, to a ray that reflects on the core/cladding interface along the propagation in the fiber. A formal derivation of the eikonal equation from the 3D Helmholtz equation is given in [11] and justifies the analogy of our system with a chaotic billiard. Indeed, the 


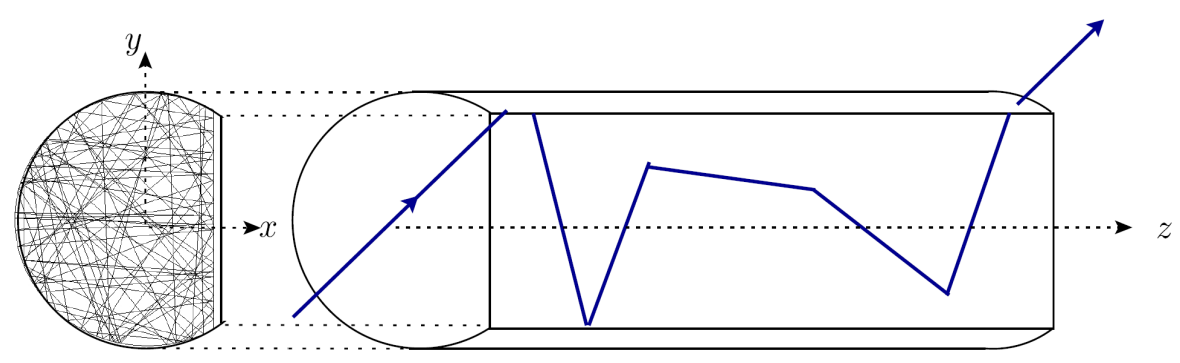

Fig. 2. Light propagation along the fiber in the geometrical limit of rays.

evolution of a ray along the fiber (in the direction of $z$ ) can be associated to a time evolution on the transverse cross-section of the fiber (see Fig. 2).

The so-called D-shaped billiard, as soon as the truncated diameter is larger than half the radius, has been proved to be a fully chaotic billiard by Bunimovich [12] and Ree and Reichl [13]. If the optical fiber can be seen as a wave billiard, it also constitutes an interesting analogous quantum system. Indeed, the propagation of the field along the fiber is described by the Helmholtz equation. In the weak guidance regime, i.e. for a small difference of indices between the core and the cladding $\left[\left(n_{\text {co }}-n_{\text {clad }}\right) / n_{\text {clad }} \ll 1\right]$, a given polarization is nearly perfectly preserved along the propagation thus enabling to use the scalar form of the Helmholtz equation

$$
\left(\Delta+\partial_{z z}\right) \psi(\boldsymbol{r}, z)+n(\boldsymbol{r})^{2} k_{0}^{2} \psi(\boldsymbol{r}, z)=0
$$

with $k_{0}$ the vacuum wave number and $n(\boldsymbol{r})$ - the index of refraction in the medium. In the paraxial approximation, this equation reduces to the following form:

$$
\mathrm{i} \beta_{\mathrm{co}} \partial_{z} \phi(\boldsymbol{r}, z)=\left[-\frac{1}{2} \Delta+V(\boldsymbol{r})\right] \phi(\boldsymbol{r}, z),
$$

where $\beta_{\text {co }}=n_{\text {co }} k_{0}$ and $\phi(\boldsymbol{r}, z)$ is deduced from $\psi(\boldsymbol{r}, z)=\phi(\boldsymbol{r}, z) \mathrm{e}^{\mathrm{i} \beta_{\mathrm{co}} z}$. The paraxial approximation is associated to the propagation of light close to the optical axis and is fulfilled as soon as the angle of the guided ray is much smaller than the cutoff angle $\theta_{\max }$, which is given by $\sin \theta_{\max }=\sqrt{1-\left(n_{\text {clad }} / n_{\text {co }}\right)^{2}}$.

Equation (2) is then formally equivalent to a pseudo-time independent Schrödinger equation. As a consequence, the D-shaped fiber appears to be an ideal wave chaos paradigm of a quantum chaos experiment.

\subsection{Typical results}

The experimental setup is conceptually simple. A beam from a helium-neon laser source at $\lambda_{\mathrm{He} / \mathrm{Ne}}=628 \mathrm{~nm}$ is collimated and enlarged so that its size becomes large enough compared to the transverse size of the fiber to be in a quasi-plane wave illumination. The main parameter of the experiment is then the angle $\theta$ between the beam and the optical axis at the fiber entrance. Indeed, this angle governs the value of the transverse wave number in the fiber $\kappa_{\mathrm{t}}$ by the way of 
the simple relation $\kappa_{\mathrm{t}}=k_{0} n_{\mathrm{co}} \sin \theta$ and, as a consequence the modes that will be excited and guided in the fiber. At the output of the fiber, one can image either the near-field (NF) intensity using a microscope objective or the far-field (FF) intensity in the focal plane of a suitable lens. Typical observations associated to a value of the transverse wave number $\kappa_{\mathrm{t}}=36 / R$ are presented in Fig. 3. As diffraction occurs due to the finite aperture of the fiber, several transverse wave numbers around the main transverse wave number $\kappa_{\mathrm{t}}$ contribute so that more than one hundred modes are superposed at the output of the fiber. The NF figure shows a speckle pattern: the intensity is statistically uniformly distributed over the whole cross-section of the fiber. This figure is associated to the superposition of several modes each with a speckle-like behavior as predicted by Berry [2]. A nice experimental evidence of Berry's conjecture is given by the FF intensity figure. In this figure, the intensity tends to fill a ring, whose mean radius is centered on the input transverse wave number $\kappa_{\mathrm{t}}$ and its width is determined by the number of contributing modes. This illustrates that the field is constituted by the random superposition of a great number of plane waves each with a fixed transverse wave number but with random directions: the field of these generic so-called speckle modes can then be seen as a real Gaussian random variable.
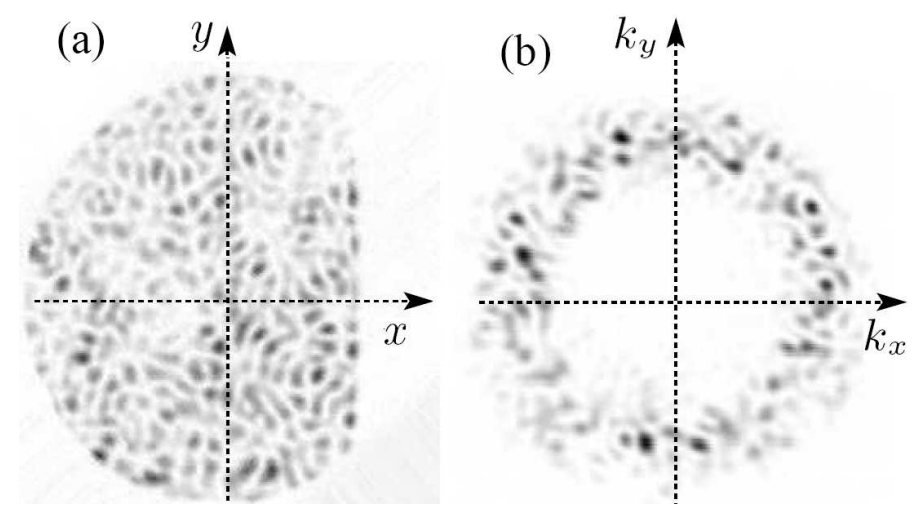

Fig. 3. Near-field intensity (a) and far-field intensity (b) for $\kappa_{\mathrm{t}}=36 / R$.

If the speckle modes are the generic observed modes, we have also experimentally investigated the series of scar modes associated to the shortest unstable periodic orbit that is the 2-bounce periodic orbit along the axis of symmetry of the fiber's cross-section. In the optical context, scar modes along the 2-bounce periodic orbit (PO) can be described as the modes of an unstable dielectric Fabry-Perot cavity. One can expect to observe a constructive interference effect building upon this periodic orbit if the following quantization condition for the value of the transverse wave number is fulfilled: 


$$
\kappa_{\mathrm{t}} \mathcal{L}=2 \pi p+\frac{\pi}{2}+\Delta \varphi
$$

where $\mathcal{L}$ is the length of the periodic orbit, $p$ - an integer, $\pi / 2$ is a phase shift due to the existence of a self focal point on the 2-bounce periodic orbit, and $\Delta \varphi$ is the phase shift due to reflection at the core/cladding interface.

By solving Eq. (3), one obtains quantized values of the transverse wave number associated to scar eigenmodes along the 2-bounce PO. For a very precise illumination launch along the direction of the 2-bounce PO with an angle associated to the wave number of the scar mode of order $p=4$, we have successfully excited a few modes around the scar mode. The NF and FF intensity observed at the output of the fiber are presented in Fig. 4.
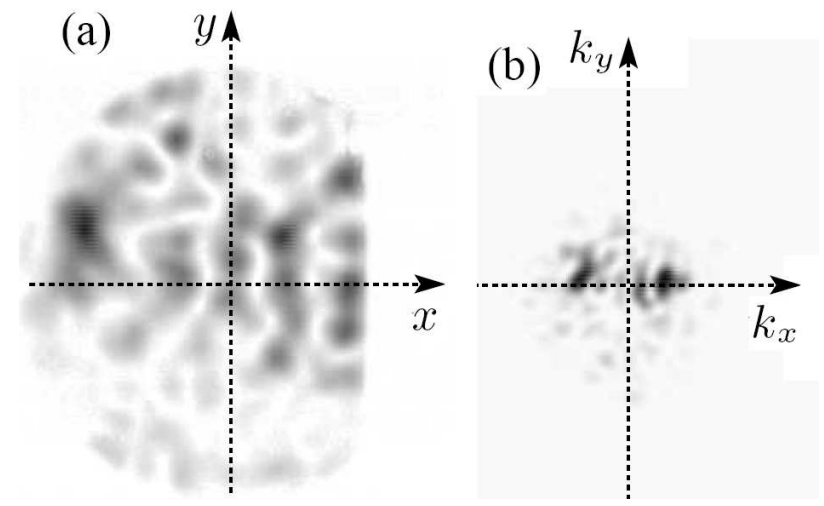

Fig. 4. Near-field intensity (a) and far-field intensity (b) associated to a superposition of a few modes around a scar mode of order $p=4$ with $\kappa_{\mathrm{t}}=10.35 / R$.

Even if several modes are excited (around thirty modes), the intensity tends to localize along the direction of the 2-bounce PO emphasizing the main contribution of the $p=4$-scar mode in the superposition. In Fig. 4b two symmetric peaks are observed which show that mainly one transverse wave number propagates along a well-defined direction, i.e. the direction of the 2-bounce PO.

\section{Selection of individual optical scars}

The previous results have revealed the optical fiber as an ideal system to study the spatial behavior of waves in a chaotic billiard. Nevertheless, the plane wave illumination condition does not permit one to excite only one mode of the fiber due to the ineluctable diffraction induced by the finite aperture of the fiber. As a consequence, looking for an individual scar mode appears to be a hard task. A mechanism of modes selection can be found in some recent investigations in the field of wave propagation in disordered media. Numerical simulations have shown that the lasing modes of a random laser can be the localized modes of the passive disordered system [14]. The lasing effect on a given localized mode is 
controlled by localizing the gain on the selected mode. Resting on these results, we propose to use a localized gain to perform a selection of the scar modes. If a passive optical fiber with a D-shape constitutes a powerful experimental system for studying wave chaos, multimode D-shaped fibers are also above all widely used to achieve optical power amplifiers [15] in which a telecom signal (called the signal) is amplified at the cost of an ancillary signal (called the pump). Usually the optical index of the erbium or ytterbium doped-core is higher than the index of the surrounding D-shaped cladding. The signal propagates in a single mode doped core, and, by guiding the pump, the multimode fiber only plays the role of an energy reservoir. By some appropriate modifications of the fabrication process, it is possible to obtain a negligible index mismatch between the small active region in the multimode fiber ${ }^{\dagger}$, at least sufficiently low to ensure that no guided mode exists inside the doped area. Then, the signal and the pump propagate on the modes of the entire section of the multimode D-shaped fiber. The crucial point is the location of the doped area: to ensure an efficient amplification of the 2-bounce scar modes, the overlap of the doped region with the scar modes has to be maximized. We choose to introduce the doping element on the self-focal point of the 2-bounce PO that also corresponds to a maximum of intensity for most of the 2-bounce PO scar modes. In the following, we present realistic numerical simulations of such a chaotic fiber with an ytterbium-doped region. The simulation is based on the beam propagation method (BPM) we already used successfully for simulating optical amplification [16]. We show how localized gain may selectively amplify a family of scar modes.

In this kind of system, optical amplification occurs due to the transfer of energy from an optical pump wave at the wavelength, $\lambda_{\mathrm{p}}=980 \mathrm{~nm}$ in our case, to the signal to be amplified at the wavelength $\lambda_{\mathrm{s}}=1020 \mathrm{~nm}$. Ytterbium is a four-level system that permits one to use different wavelengths for the pump and the signal preventing direct signal reabsorption. As a consequence, ytterbium in a silica matrix can be treated as a three energy-level system with a metastable third energy level.

Using the evolution equations of the population density, one can easily obtain the following expressions for the evolution of the pump and signal intensities [17]:

$$
\begin{aligned}
\frac{\mathrm{d} I_{\mathrm{p}}}{\mathrm{d} z} & =-\sigma_{\mathrm{pa}} N_{1}(z) I_{\mathrm{p}}(z), \\
\frac{\mathrm{d} I_{\mathrm{s}}}{\mathrm{d} z} & =\sigma_{\mathrm{sa}}\left(\eta_{\mathrm{s}} N_{2}-N_{1}\right) I_{\mathrm{s}}(z),
\end{aligned}
$$

where $I_{\mathrm{p}}$ and $I_{\mathrm{s}}$ are, respectively, the intensity of the pump and of the signal, $\sigma_{\mathrm{sa}}$ - the absorption cross-section of the signal, $\sigma_{\mathrm{pa}}$ - the absorption cross-section for the pump and $\eta_{\mathrm{s}}=\sigma_{\mathrm{se}} / \sigma_{\mathrm{sa}}$, where $\sigma_{\mathrm{se}}$ is the emission cross-section of the signal. As soon as the intensity of the signal $I_{\mathrm{s}}$ is low compared to the intensity of the

${ }^{\dagger}$ The actual fiber has been fabricated in our institute by W. Blanc, in collaboration with P. Oupicky from Plasma Institute of Physics in Turnov (Czech Republic). 
pump $I_{\mathrm{p}}$, Eq. (4) only depends on $I_{\mathrm{p}}$ and the two equations are partially coupled so that they can be treated separately. This case is fulfilled in our simulation, where the initial signal power $P_{\mathrm{s}_{0}}=100 \mathrm{~mW}$ and the pump power equals to $P_{\mathrm{p}_{0}}=6 \mathrm{~W}$.

The BPM algorithm is modified to take Eqs. (4), (5) into account to simulate the coupled evolutions of the pump and the signal. To make an efficient transfer of energy from the pump to the signal, one has to maximize the number of excited pump modes: a focused laser beam at the pump wavelength is therefore launched in the multimode core. A great number of modes of the D-shaped core are thus excited and the overlap of their field with the doped area is responsible for the energy transfer from the pump to the signal. In previous works, we have shown that the chaotic D-shaped geometry is an efficient shape for this purpose [18]. The evolution of the pump along the fiber follows an early exponential decay due to absorption in the doped area that tends to saturate as soon as the pump power is no longer able to fulfill the population inversion condition $\eta_{\mathrm{s}} N_{2}-N_{1}>0$.

\section{Results}

We first study the influence of the doped area for the initial illumination used to perform the experimental observation of Fig. 4. We simulate a plane wave illumination in the direction of the 2-bounce PO with a transverse wave number associated to the scar mode of order $p=4$. In Fig. 5, we plot the evolution of the power of the signal along the propagation that clearly shows that the signal is getting amplified along the propagation. The maximum of amplification (the propagation length $z_{\max }$, for which the gain of the signal is a maximum) is obtained for $z_{\max }=41 \mathrm{~m}$.

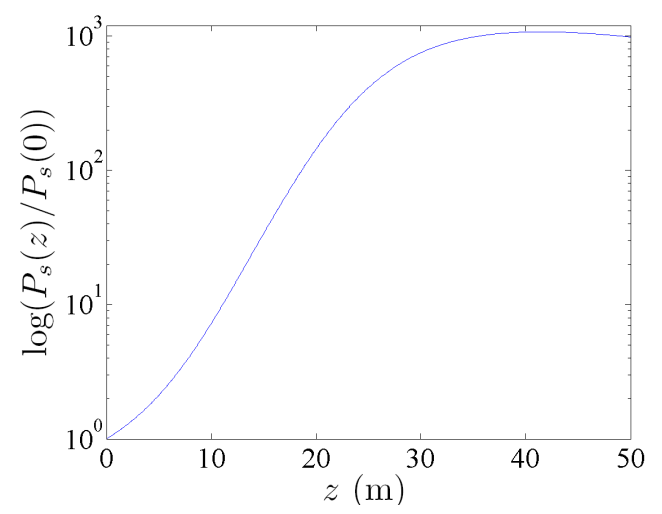

Fig. 5. Evolution of the signal power along the propagation.

The near-field and far-field patterns observed at this propagation length are reported in Fig. 6. The NF intensity is very similar to the calculated scar mode of order $p=4$. The dominating contribution of the $p=4$-scar mode is emphasized in the FF intensity: two symmetrical peaks centered on the value of the wave 


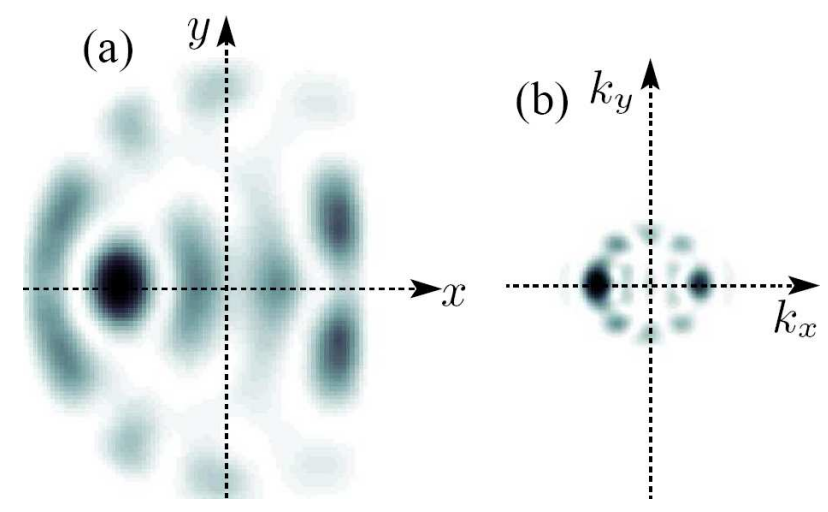

Fig. 6. NF (a) and FF (b) at the length of the maximum amplification.

number associated to the scar mode of order $p=4$ appear. Mainly one direction and value of the transverse wave number are selected.

To clearly identify the guided modes along the propagation, we calculate what we call a pseudo-spectrum of our system. This pseudo-spectrum is derived from a standard semiclassical procedure developed by Tomsovic and Heller [19] and adapted to our numerical algorithm [20]. The pseudo-spectrum is extracted from the Fourier transform of the correlation function $C(z)$ obtained from the overlap of the initial field $\psi_{0}(\boldsymbol{r})$ with the propagating field $\psi(\boldsymbol{r}, z)$. We follow the evolution of the pseudo-spectrum along the propagation by applying the previous method to shifted windows of length $Z=1.3 \mathrm{~m}$ evaluated each $0.65 \mathrm{~m}$. Figure 7 shows the pseudo-spectrum calculated at the input of the fiber, and around the length of the maximum gain, $z_{\max }$.
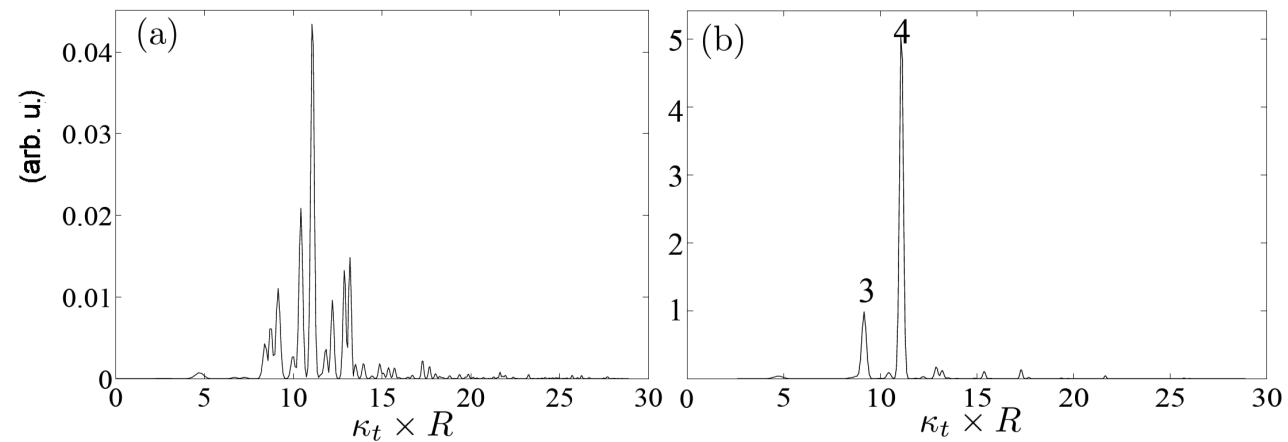

Fig. 7. Pseudo-spectra evaluated at the input of thie fiber (a) and at the length of maximum amplification (b).

The initial pseudo-spectrum is centered on the transverse wave number $\kappa_{p=4}$ and broadened due to the diffraction at the fiber entrance. At length $z_{\max }$, the broadening of the pseudo-spectrum due to diffraction seems to be limited by the 
influence of the localized gain that induces a mechanism of selective amplification of the selected scar. Only the scar modes of order $p=3$ and $p=4$ initially excited are amplified along the propagation. To check the robustness of this scar modes selection process, we launch a spatially incoherent initial field, that is, a field resulting from a superposition of transverse wave vectors with random directions and random moduli up to $\kappa_{\mathrm{t}}=33 / R$. The field propagates along the fiber and we observe the evolution of the far-field. Figure 8 shows three pictures of the far-field intensity: at the entrance of the fiber, after $20 \mathrm{~m}$, and for the maximum gain length $z_{\max }$.

(a)

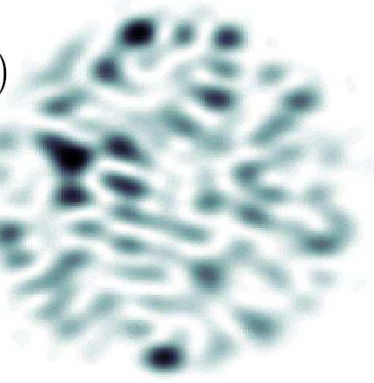

(b)

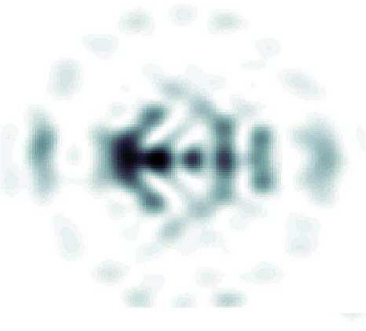

(c)

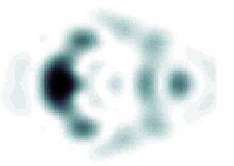

Fig. 8. Initial incoherent FF (a), after $20 \mathrm{~m}$ (b) and for the length corresponding to the maximum of amplification (c).

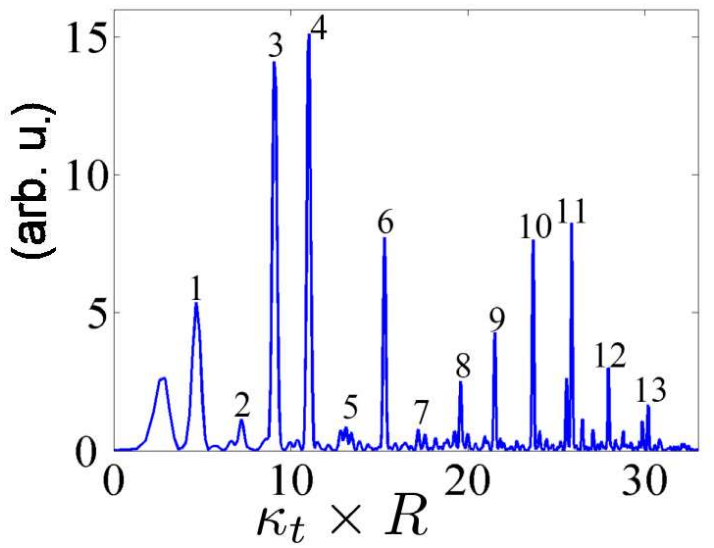

Fig. 9. Pseudo-spectrum evaluated at the maximum amplification length for an initial spatially incoherent field.

Along the propagation, we observe a clear selection of the direction of the transverse wave number along the 2-bounce PO so that for the maximum gain length only two symmetric spots dominate the FF pattern. Therefore, the spatial 
incoherent field tends to condensate on a specific value of the transverse wave number leading to a coherent field.

The pseudo-spectrum associated to $z_{\max }$ (Fig. 9) shows a succession of quasi-periodic peaks. The value of the transverse wave number of each peak corresponds to the quantified value of transverse wave number for each scar mode up to $\kappa_{\mathrm{t}}=33 / R$. All the scar modes have been selected and amplified thanks to the localized gain. The weight of each peak is directly linked to the overlap of the scar modes with the doped area: scar modes of order 2, 5, and 7 have indeed a bad overlap with the doped area, whereas the scar mode of order $p=4$ has the best overlap. From these results, we can conclude that the localized gain acts as a scar filter.

\section{Conclusion}

The optical fiber with a truncated cross-section appears to be a good candidate to image experimentally wave chaos: complementary information about the field that propagates in the chaotic billiard are deduced from the NF and FF intensity. The ergodic behavior of generic modes has been proved experimentally and scar modes have first been observed in an optical fiber. Due to the inescapable diffraction at the fiber input, the properties of individual scar modes cannot be easily studied experimentally. To perform a selective excitation of scar modes in our multimode structure, we have investigated the influence of a localized gain area on the propagation of a plane-wave illumination. Our numerical results clearly show that scar modes are selectively amplified. The experimental validation is in progress and will be the subject of a next publication.

\section{Acknowledgments}

We would like to thank the Active Optical Fiber group of the LPMC for the realization of our "exotic" preform and the undergraduate and master students A. Martin and S. Onteniente for their contribution to this work. This research is part of the project Chaos On Line No. ANR-05-JCJC-0099-01 supported by the National Agency of Research (ANR).

\section{References}

[1] E.J. Heller, Phys. Rev. Lett. 53, 1515 (1984).

[2] M.V. Berry, Proc. R. Soc. A 423, 219 (1989).

[3] E. Bogomolny, Physica D 31, 169 (1988).

[4] L. Kaplan, E.J. Heller, Phys. Rev. E 59, 6609 (1999).

[5] H.-J. Stöckmann, Quantum Chaos: An Introduction, Cambridge University Press, Cambridge 1999.

[6] J. Stein, H.-J. Stöckmann, Phys. Rev. Lett. 68, 2867 (1992).

[7] A. Kudrolli, Mathew C. Abraham, J.P. Gollub, Phys. Rev. E 63, 026208 (2001). 
[8] A. Mekis, J.U. Nockël, G. Chen, A.D. Stone, R.K. Chang, Phys. Rev. Lett. 75, 2682 (1995).

[9] V. Doya, O. Legrand, F. Mortessagne, C. Miniatura, Phys. Rev. Lett. 88, 014102 (2001).

[10] M.V. Berry, J. Phys. A, Math. Gen. 10, 2083 (1977).

[11] V. Doya, O. Legrand, F. Mortessagne, C. Miniatura, Phys. Rev. E 65, 056223 (2002).

[12] L.A. Bunimovich, Commun. Math. Phys. 65, 295 (1979).

[13] S. Ree, R.E. Reichl, Phys. Rev. E 60, 1607 (1999).

[14] P. Sebbah, C. Vanneste, Phys. Rev. B 66, 144202 (2002).

[15] E. Desurvire, Erbium Doped Amplifier, Wiley Interscience, New York 1994.

[16] V. Doya, O. Legrand, F. Mortessagne, Opt. Lett. 26, 872 (2001).

[17] A. Ghatak, K. Thyagarajan, Introduction to Fiber Optics, Cambridge University Press, Cambridge 1998.

[18] P. Leproux, V. Doya, P. Roy, D. Pagnoux, F. Mortessagne, O. Legrand, Opt. Commun. 218, 249 (2003).

[19] S. Tomsovic, E.J. Heller, Phys. Rev. E 47, 1 (1993).

[20] M.D. Feit, J.A. Fleck, Appl. Opt. 19, 1154 (1980). 